\title{
Research on the Air Quality Evaluation and Regional Interaction in Jing-Jin-Ji Region
}

\author{
Wentao Feng \\ School of Mechanical Engineering, North China Electric Power University, Baoding 071003, China;
}

fengwentao9502@163.com

Keywords: Air quality evaluation Regional interaction Jing-Jin-Ji region coordinated development

\begin{abstract}
The problem of air pollution has attached people's general attention nowadays. And the Jing-Jin-Ji region is badly polluted in particular. So this paper will mainly focus on how to evaluate the air quality and regional interaction in Jing-Jin-Ji region in a new scientific approach. Considering that the existing air quality evaluation methods have some weakness such as the single evaluation factor and the air pollution is regional, an air quality evaluation model based on the principle component analysis is developed in this paper firstly to set up a new comprehensive evaluation index which can evaluate the air quality more scientifically and more reasonably. Then, a regional interaction model is developed based on the gravity model and the analogy method to evaluate regional interaction in Jing-Jin-Ji region. The result and analysis are shown at the end of this paper. Finally, effective strategy can be put forward to solve the dreadful air pollution in this region and promote Jing-Jin-Ji coordinated development.
\end{abstract}

\section{Introduction}

\subsection{Background}

As is known to all, the coordinated development of Beijing-Tianjin-Hebei, which is customarily called Jing-Jin-Ji region, has become a current hot topic in China. And it is a major strategic decision made by the CPC central committee and the state council to promote coordinated development of the Jing-Jin-Ji region. However, the air pollution has become a critical bottleneck restricting the regional development. Controlling air pollution and improving the ecological environment has become an important breakthrough to promote coordinated development. So it is of prime significance to research on the evaluation and regional interaction, which can actively help to take the lead in progress on the coordinated control of air pollution in Jing-Ji-Jin region and promote coordinated development in return.

\subsection{What the paper do:}

To research on the air quality, a new air quality evaluation index based on the principle component analysis is proposed in this paper first, which are proved to be more scientific and reasonable than the AQI. Then a regional interaction model is based on the Gravity Model to study on the mutual influence among the region. At last, the air quality evaluation index and the regional interaction can be available. Then sensible proposals are given based on the results to solve the problem of air pollution in Jing-Jin-Ji region more efficiently.

In this paper, we choose all the 10 cities in the region to analyze. And all the statistics are drawn from Ministry Environmental Protection of China at http://www.mep.gov.cn/zwgk/hjtj/.[1]

\section{The Air quality evaluation Model}

\subsection{Application of principal component analysis [2]}

Considering that the statistical data is given for many types of pollutants, some of which have little impact on local air quality, the principal component analysis is used to reduce the dimension of pollutants so as to make the best comprehensive simplification in the principle of ensuring the least loss of information. 
The results of component analysis can be reached through computation, and the first three components are selected as the principal components, the eigenvalue of which are greater than one and the total cumulative rate of which are greater than 80 percent.

Some partial results can be shown in Table1. to Table3.

Table1 Principal Component analysis result of Beijing City

\begin{tabular}{cccc}
\hline Component number & Eigenvalue & Contribution rate & Total Cumulative rate \\
\hline 1 & 8.512 & $65.477 \%$ & $65.477 \%$ \\
2 & 2.015 & $15.651 \%$ & $81.128 \%$ \\
3 & 1.123 & $8.636 \%$ & $89.764 \%$ \\
\hline
\end{tabular}

Table2 Principal Component analysis result of Tianjin City

\begin{tabular}{cccc}
\hline Component number & Eigenvalue & Contribution rate & Total Cumulative rate \\
\hline 1 & 8.296 & $63.812 \%$ & $63.812 \%$ \\
2 & 2.465 & $18.960 \%$ & $82.773 \%$ \\
\hline
\end{tabular}

Table3 Principal Component analysis result of Shijiazhuang City

\begin{tabular}{cccc}
\hline Component number & Eigenvalue & Contribution rate & Total Cumulative rate \\
\hline 1 & 7.874 & $60.568 \%$ & $60.568 \%$ \\
2 & 2.665 & $20.503 \%$ & $81.070 \%$ \\
3 & 1.005 & $7.733 \%$ & $88.803 \%$ \\
\hline
\end{tabular}

Similarly results of other 7 cities can be get. The results show that the pollutant index in each can be instead by two or three principal components, largely reducing the dimension of the statistical data.

\subsection{The comprehensive evaluation index based on principal component analysis}

The existing air quality evaluation methods have some weakness such as the single evaluation factor, the lack of national standard, which cannot adapt to the needs of environment protection work well. So a new index to evaluate the air quality is developed based on the principal component analysis and comprehensive evaluation index as follows.

The new index takes both the principal component ant its contribution rate into consideration, which can be written as follows.

$$
\mathrm{Q}=\sum_{\mathrm{i}=1}^{\mathrm{n}} \mathrm{t}_{\mathrm{i}} \times \delta_{\mathrm{i}}
$$

In which the $\mathrm{Q}$ is the comprehensive evaluation index; $t_{i}$ is the $T$-score of the principal component $\mathrm{i} ; \delta_{\mathrm{i}}$ is the contribution rate of the principal component $\mathrm{i}$;

The calculation method of T-score is as follows: [3]

First, performing principal component analysis by the SPSS software to get the component score coefficient matrix; then, transforming it into a feature vector matrix; last, getting the T-score through a $\mathrm{T}$ fractional transformation.

Taking Beijing for example, the result can be shown as follows in Table4.

Table4. Comprehensive evaluation index result of Beijing City

\begin{tabular}{ccccc}
\hline Date & $\mathrm{t}_{1}$ & $\mathrm{t}_{2}$ & $\mathrm{t}_{3}$ & $\mathrm{Q}$ \\
\hline 1 & 30.62 & 33.16 & 33.78 & 28.16 \\
2 & 50.68 & 44.87 & 55.80 & 48.56 \\
3 & 85.17 & 59.56 & 82.68 & 72.23
\end{tabular}

The air quality of every city can be evaluated by the comprehensive evaluation index above. The new method to evaluate air quality, which takes the major pollutants and their pollution level into consider, is not only scientific and reasonable but also more applicable to the environment protection work. 


\section{The Regional interaction Model}

\subsection{The introduction of the Gravity Model}

The gravity model is a mathematical model which is widely used to analyze the spatial interaction, that is, it can be applied to analyze and predict the spatial interaction. Theoretically, the interaction between two cities is positively related to the urban air quality while negatively correlated with the distance between them. But it is only an ideal state of potential energy. In fact, the interaction can be affected by many factors. Considering the complexity of the weather, the inner-city wind speed and the geographical position, the gravity model is introduced to be a index to measure the interaction.

The gravity model is based on the distance decay principle and the principle of gravitation, which can be described as follows:

$$
I_{i j}=G \cdot M_{i} \cdot M_{j} / D_{i j}^{2}
$$

Where $I_{i j}$ is the interaction between two cities; $M_{i}, M_{j}$ are the weight of city i and city $j$; $D_{i j}$ is the distance between city $\mathrm{i}$ and city $\mathrm{j}$; $\mathrm{b}$ is the distance coefficient of friction. The value of $\mathrm{G}$ and $\mathrm{b}$ are based on the actual situation accordingly in different regions. Generally, $G=1, b=2$.

\subsection{The Regional interaction Model based on the Gravity Model}

When it comes to the interaction in air quality between different cities, it can be easily found that the change of air quality in two adjacent cities has a strong joint action. So it can be considered that there is a great correlation between them to some extent. That is, the greater the product of the comprehensive evaluation index of air quality between the two cities, the stronger the interaction in air quality is; the greater the distance between the two cities, the greater the space distance of impedance is, which means that the interaction between them is reduced with the increase of the distance.

Then the regional interaction model is developed with analogy to the Gravity Model. The gravity model is modified to get regional interaction model as follows:

$$
\left\{\begin{array}{c}
F_{i j}=Q_{i} \cdot Q_{j} / D_{i}^{2} \\
\mu=F_{i j} / \sum_{j=1}^{n} F_{i j} \\
F_{i}=\sum_{j=1}^{n} F_{i j}
\end{array}\right.
$$

Where $F_{i j}$ is the degree of regional interaction, $\mu$ is the membership grade between city $i$ and city j; $F_{i}$ is the total external impact of city $i$.

We can get the effect of each city to another city through the above model, and the interaction in air quality can be made out. The result of the application of the model to Jing-Jin-Ji region can be shown as follows in Fig.1 


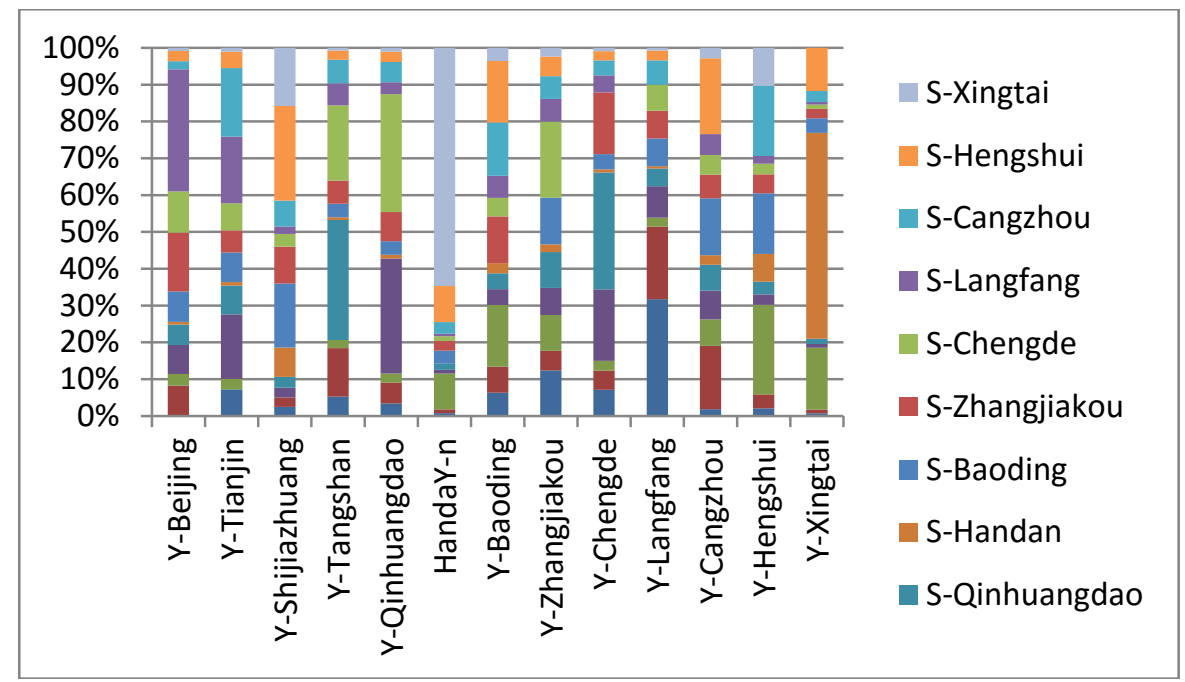

Fig.1 The interaction of air quality in Jing-Jin-Ji region in which the city with a prefix ' $\mathrm{Y}$ ' means it is the pollution sources and a ' $\mathrm{S}$ ' means the pollution receptor, and each city is both a sources and a receptor. All the 13 cities are from the Jing-Jin-Ji region

\section{Conclusion}

From the analysis above, we can get to the conclusion that the 10 cities in Jing-Jin-Ji region have close contacts; each city has an impact on any other 9 cities, while Handan and Xingtai have a great effect to each other. We can know about the interaction of air quality in Jing-Jin-Ji region, which give help to make rational decision to promote coordinated development in this special region as follows.

- There is a certain degree of regional interaction in air quality among the 13 cities of Jing-Jin-Ji region, that is, it is necessary to strengthen the regional joint prevention and control measure to improve the overall level of air quality in this region.

- According to the results, the interaction between Handan and Xingtai is stronger, so is the interaction between Beijing and Langfang, so it is proposed that the four cities should be paid more attention to in $\mathrm{t}$ the regional joint prevention and control measure.

- Establishment of a reasonable evaluation index of quality and establishment of regional cooperation are of much importance.

Furthermore, the model presented in this paper can be applied to any other regional analysis on the issue of environment, which can give help to regional coordinated development.

\section{References}

[1] Information on http://www.mep.gov.cn/zwgk/hjtj/.

[2] Xie.L., Song.ZG. Spss statistical analysis tutorial, second ed., Beijing: Posts and Telecommunications Press.

[3] Xie. L., Song.ZG. Spss statistical analysis from entry to master, Beijing: Tsinghua University Press.

[4] Wu. XW. Research on the economic spatial relations and spatial structure of the Central Plains Economic Zone Based on the gravity model, Shaoxing: Journal of Shaoxing University.

[5] Xie. L., Song.ZG. Spss statistical analysis from entry to master, Beijing: Tsinghua University Press.

[6] Zhang. J., Miao.H., Ouyang.ZY., Wang.XK. Analysis on the change of air quality and its influencing factors in the suburban area of Beijing city in recent 20 years, Beijing: Journal of environmental sciences. 